\title{
Research on Map Construction and Location of Laboratory Service Robot based on Iterative Closest Point
}

\author{
Jian Wu, Ruting Yao, Yili Zheng *, Jinhao Liu
}

\begin{abstract}
The development of mobile robots has led to their wide application in a variety of fields. This study focuses on the intelligent application of mobile robots in laboratory management, especially the environmental awareness and self-positioning of a robot in the laboratory. In this study, a wheeled mobile robot is selected and equipped with a 2D laser scanner. Based on this, a Robot Operating System (ROS) environment is built. The nearest neighbor iterative closest point (ICP) matching algorithm is utilized to perceive the laboratory service environment, construct the indoor map in real time, and locate the robot precisely. Subsequently, data collected in the corridors and indoor environment of the experimental building are used to test the accuracy of the ICP matching algorithm. The results showed that the minimum translation error is as low as $0.0003 \mathrm{~m}$ and that the minimum rotation angle error is less than $0.5^{\circ}$. In addition, the positioning and mapping of the robot were analyzed. The experimental results show that the ICP matching algorithm is well suited to map construction and positioning of the laboratory service robot. This is of great significance for further research on laboratory service robots.
\end{abstract}

Keywords-ICP algorithm ; SLAM ; Service robot

\section{INTRODUCTION}

$\mathrm{W}$ ith the rapid development of the robotics industry, service robots have become widely used in all walks of life. As an important location for experimental teaching and technology research, the laboratory requires a large amount of manpower to complete monotonous and repetitive work such as teaching, equipment sorting, information recording and so on. A service robot, which is a type of high-efficiency automation equipment, can effectively solve those problems in laboratory applications. This paper describes a laboratory service robot based on a Robot Operating System (ROS) as the control platform that obtains indoor environmental information through a 2D laser scanner, and establishes a task of simultaneous localization and mapping. 2D codes were pasted on the experiment cabinet and equipment to access the equipment information, and a monocular camera was used to obtain the information stored by the 2D code.

This work was supported in part by the National Natural Science Foundation of China (NO.31670719).

Jian Wu, Ruting Yao, Yili Zheng, Jinhao Liu are with the Beijing Forestry University, Beijing 100083, China. (corresponding author phone: 010-62336922; e-mail: zhengyili@bjfu.edu.cn).

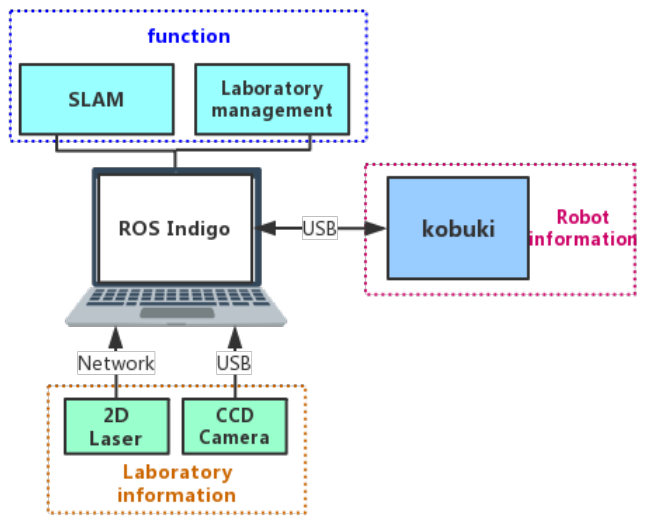

Fig. 1. Architecture of laboratory Service Robot

As an indoor service robot, the laboratory service robot needs some key technologies to realize autonomous work in complex environments, including positioning, navigation, identification and interaction. Among them, the laboratory service robot's judgment of the laboratory environment and its estimation of its own location are the primary foci of this research [1][2]. Because it provides a prerequisite for the robot to achieve other functions, such as avoiding obstacles, moving to target points, planning paths, and automatic charging. It is also the core technology of autonomous mobile robots and the key to achieving intelligence. Thus, we introduce simultaneous localization and mapping based on laser data (Laser SLAM). The core part of Laser SLAM is the head match. The data is matched according to the data measured by the external sensor. The main function of the head match is pose prediction, which refers to the estimation of the robot's current posture by using the relationship between the known previous posture and the current observed value, and the pose of robot are obtained [3]. The detection information of the current position of the robot is matched with the known map to accurately obtain the position of the robot [4]. The iterative closest point (ICP) algorithm is the most widely used point cloud registration algorithm, which can meet the precision requirements of point cloud registration. Therefore, the ICP algorithm is a good choice for data matching in Laser SLAM for the head match.

The ICP algorithm was proposed by Bsel and Mckey to solve the registration problem of $3 \mathrm{D}$ point clouds [5]. Since then it has been continuously improved by researchers and widely used in robot research. Holz and 
Behnke in [6] proposed an effective combination SLAM algorithm based on the ICP algorithm in a dynamic environment. Peng extracted the line segments from the laser data and fitted them, then used the ICP algorithm to scan and match the laser front and back frame scan data, so as to correct the odometer information [7]. Tiar tested two variants of ICP-SLAM in Robucar, a dual-steering robot, and achieved good results [8]. In [9], the application of the Trimmed ICP method, corner feature matching based HAYAI algorithm and point-line matching based the point-to-line ICP (PL-ICP) method in an indoor SLAM were explored, and their applicable scopes were given. In [10], Djehaich et al. proposed a Boolean-based ICP-SLAM method, which makes it easier to find rigid transformations. In order to eliminate discrete points in the scanned data, Liu proposed an ICP matching scanning method based on continuous lattice sequences, which reduces the correspondence between extreme distances and improves the matching accuracy [11]. In [12], researchers have proposed a SLAM that combines two matching methods of ICP and feature points in an indoor environment. Yang et al. proposed a self-localization method based on filtered ICP (FICP) for solving the robot positioning problem in the ICP algorithm [13].

This paper focuses on the effectiveness of the ICP algorithm in the operation of laboratory service robots. In terms of practicality, the accuracy and efficiency of the ICP algorithm for environmental awareness and self-positioning of laboratory service robots are described in detail. The robot experiment platform consists of a wheel robot and a 2D laser scanner operating under the ROS system. The scan data in the actual laboratory environment is analyzed, and the matching results between the two frames before and after are obtained to perform the pose prediction. Finally, a grid map is used to construct a map of the experimental environment, and the positioning problem of the robot is tested.

\section{ICP ALGORITHM}

$\mathrm{T}$ he ICP algorithm is a mature scan-matching algorithm. The principle is to select the target point cloud $\mathrm{P}$ and source point cloud $\mathrm{X}$, find the nearest point (pi, xi) according to certain constraints, and then calculate the rigid transformation (rotation matrix $\mathrm{R}$ and translation matrix $\mathrm{t}$ ) between them. The error is as follows:

$$
e_{i}=x_{i}-\left(R * p_{i}+t\right) \text {.\#(1) }
$$

By constructing the least square equation, the error function $E(R, \mathrm{t})$ were obtained:

$$
E(R, t)=\frac{1}{N_{p}} \sum_{i=1}^{N_{p}}\left\|x_{i}-R p_{i}-t\right\|^{2} \#(2)
$$

The algorithm flow is as follows:

(1) The point set $p_{i}$ is selected in the target point cloud $P$, and the corresponding point set $x_{i}$ is selected in the source point cloud $X$.

Q The centroid of the target point cloud and the source point cloud is defined as

$$
u_{x}=\frac{1}{N_{p}} \sum_{i=1}^{N_{p}} x_{i}, u_{p}=\frac{1}{N_{p}} \sum_{i=1}^{N_{p}} p_{i} \#(3)
$$

and the centroid coordinates are obtained

$$
\mathrm{X}_{i}^{\prime}=\left\{x_{i}-u_{x}\right\}, \mathrm{P}_{i}^{\prime}=\left\{p_{i}-u_{p}\right\} \#(4)
$$

(3) $\mathrm{R}$ and $\mathrm{t}$ are computed by singular value decomposition, assuming

$$
W=\sum_{i=1}^{N_{p}} x_{i}{ }^{\prime}{ }_{i}^{T}=U \sum V^{T} \quad \#(5)
$$

the rotation matrix $\mathrm{R}$ and translation matrix $\mathrm{t}$ are

$$
R=V U^{T}, t=u_{x}-R u_{p} \#(6)
$$

(4) A new set of points is obtained,

$$
p_{i}^{*}=R p_{i}+t \#(7)
$$

and the average distance $\mathrm{d}$ from $p_{i}^{*}$ and $x_{i}$ is calculated by (2).

(DIf $d$ is less than the preset threshold or the number of iterations is more than the maximum, it will stop and jump to step $\varnothing$ until the convergence condition is satisfied [5][14-18].

The essence of the ICP algorithm is the best match based on the least square method. The ICP algorithm repeats the process of "determining the point set of the corresponding relationship, calculating the rigid body deformation and calculating the error" until the convergence criterion of the correct match is met.

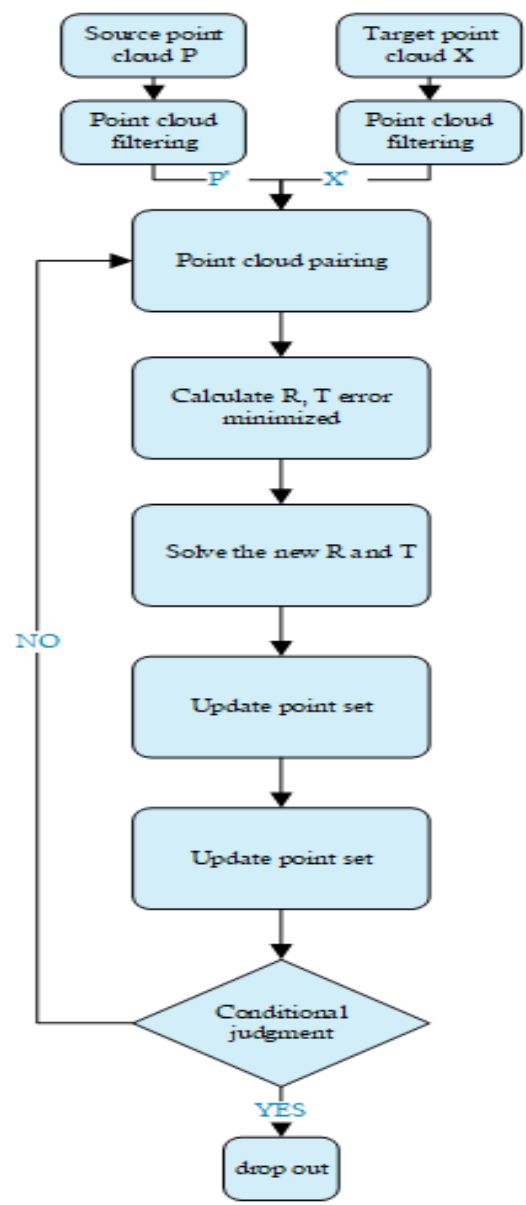

Fig. 2. ICP algorithm flow chart 


\section{ICP-SLAM ALGORITHM}

$\mathrm{T}$ he basic principle of ICP-SLAM is to match the point cloud scanned by LiDAR with the point cloud in the original map [8][18] to get the initial pose of the robot $P(x, y, \theta)$ and store it in the map. Next, the LiDAR data from the mobile robot to the next state are read, and the next pose is estimated. The predicted pose is optimized by using ICP algorithm. Then, the optimized position and posture are stored in the map to complete the map updating. These steps are repeated until the robot stops working. ICP-SLAM is mainly divided into three parts, as shown in Figure 3.

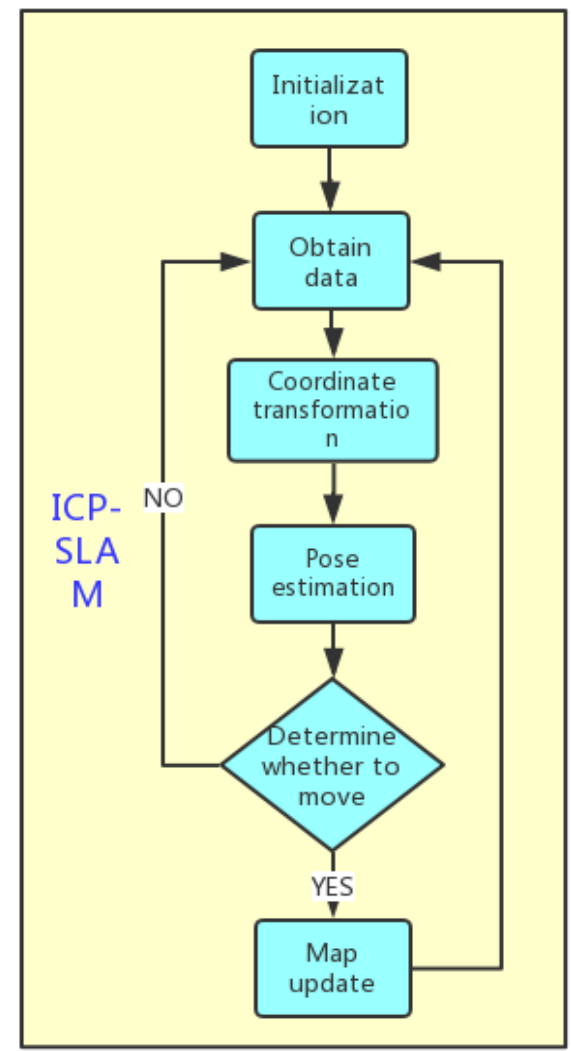

Fig. 3. ICP-SLAM algorithm

\section{A. Coordinate transformation and data association}

As shown in Figure 4, the laboratory service robot mainly includes four important coordinate systems: base_link, laser, odom and map. Among them, base link represents the robot coordinate system, odom represents the odometer coordinate system, laser represents the lidar coordinate system, and map represents the map coordinate system (global coordinate system).

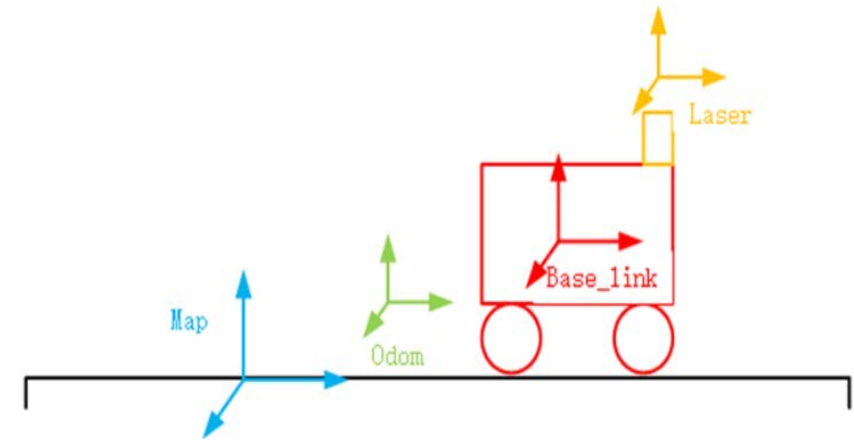

Fig. 4. The coordinate systems of robots
Coordinate transformation refers to the projection among the laser coordinate system, the robot coordinate system and the world coordinate system.

For example, a co ordinate system $C_{r e f}$ is defined as the robot coordinate system, and the LiDAR data $C_{\text {new }}$ is projected into $C_{\text {ref }}$, and $C_{\text {new }}^{\prime}$ is obtained. The conversion relationship is as follows:

$$
C_{\text {new }}^{\prime}=R_{l} * C_{\text {new }}+t_{l} \text {.\#(8) }
$$

In the equation,

$$
R_{l}=\left[\begin{array}{cc}
\cos \theta & -\sin \theta \\
\sin \theta & \cos \theta
\end{array}\right] \#(9)
$$

is the rotation matrix, $\theta$ is the angle between the laser coordinate system and the robot coordinate system, and

$$
t_{l}=\left[\begin{array}{l}
t_{x} \\
t_{y}
\end{array}\right] \#(10)
$$

is the translation matrix, where $t_{x}$ and $t_{y}$ are the differences in the direction of $\mathrm{X}$ and $\mathrm{Y}$ axes respectively.

The data points in the two coordinate systems are correlated, and the minimum Euclidean distance $d$ between them is calculated. When $d$ is the minimum, the two data points are the associated data points.

\section{B. Posture estimation and positioning}

Posture estimation is based on data association, calculating the difference $\left[\begin{array}{lll}t_{x} & t_{y} & \theta\end{array}\right]$ between the two frames of laser data, thus predicting the current position and motion of the robot, and verifying the accuracy of the prediction by minimizing the error [8]. The error function is as follows:

$$
\begin{gathered}
\mathrm{J}=\frac{1}{\mathrm{~N}} \sum_{\mathrm{i}=1}^{\mathrm{N}}\left[\left(\mathrm{X}_{\text {ref }}(\mathrm{c}(\mathrm{i}))-\mathrm{X}_{\text {new }}^{\prime}(\mathrm{c}(\mathrm{i}))\right)^{2}+\left(\mathrm{Y}_{\text {ref }}(\mathrm{c}(\mathrm{i}))-\right.\right. \\
\left.\left.\mathrm{Y}_{\text {new }}^{\prime}(\mathrm{c}(\mathrm{i}))\right)^{2}\right]
\end{gathered}
$$

in the equation, $X_{\text {ref }}$ and $Y_{\text {ref }}$ refer to the coordinates of the laser data of the previous frame in the reference frame, $X_{\text {new }}^{\prime}$ and $Y_{\text {new }}^{\prime}$ refer to the coordinates of the laser data of the current frame in the reference frame, which are obtained by $X_{\text {new }}$ and $Y_{\text {new }}$ in equation (7).

At the same time, the feature $m$ in the laboratory environment is extracted from the laser scanning data, and the pose of $m$ in the robot coordinate system is estimated. Then the pose of $m$ in the laboratory environment coordinate system is calculated, and $m$ is added to the map. When the position and posture of the robot are changed, $m$ is estimated again. The position of the robot in the laboratory environment can be obtained according to the pose changes of $m$ in the coordinate systems of the robot and laboratory environment.

\section{Map management}

Since the grid map is easy to construct, save and express, this paper adopts the grid map. A grid map divides the environment into a series of grids that discretize the environment, each grid corresponding to a point on the map. After the robot moves for a cer tain distance, the established laboratory map should be updated in time. Its main content is to update the probability of occupancy of each grid. For each grid $m_{i}$, the assumption $p\left(m_{i}\right)>0.8$ indicates that the grid is 
occupied and $p\left(m_{i}\right)<0.2$ indicates that the grid is not occupied, $x_{t}$ refers to the position and attitude of the robot at time $t, z_{t}$ refers to the observation data at time $t$. The shape of the grid $m_{i}$ can be expressed as

$$
\mathrm{p}\left(\mathrm{m}_{\mathrm{i}} \mid \mathrm{x}_{1: \mathrm{t}}, \mathrm{z}_{1 \mid \mathrm{t}}\right)=\frac{\mathrm{p}\left(\mathrm{m}_{\mathrm{i}} \mid \mathrm{z}_{\mathrm{t}}, \mathrm{x}_{\mathrm{t}}\right) * \mathrm{p}\left(\mathrm{z}_{\mathrm{t}} \mid \mathrm{x}_{\mathrm{t}}\right)}{\mathrm{p}\left(\mathrm{m}_{\mathrm{i}}\right)} * \frac{\mathrm{p}\left(\mathrm{m}_{\mathrm{i}} \mid \mathrm{z}_{\left.1: \mathrm{t}-1, \mathrm{x}_{1: \mathrm{t}-1}\right)}\right)}{\mathrm{p}\left(\mathrm{z}_{\mathrm{t}} \mid \mathrm{z}_{\left.1: \mathrm{t}-1, \mathrm{x}_{1: \mathrm{t}}\right)}\right.} .
$$

The probability of the opposite value of the state of a grid $m_{i}$ can be expressed as

$$
\begin{gathered}
\mathrm{p}\left(\sim \mathrm{m}_{\mathrm{i}} \mid \mathrm{x}_{1: \mathrm{t}}, \mathrm{z}_{1 \mid \mathrm{t}}\right)= \\
\frac{\mathrm{p}\left(\sim \mathrm{m}_{\mathrm{i}} \mid \mathrm{z}_{\mathrm{t}}, \mathrm{x}_{\mathrm{t}}\right) * \mathrm{p}\left(\mathrm{z}_{\mathrm{t}} \mid \mathrm{x}_{\mathrm{t}}\right)}{\mathrm{p}\left(\sim \mathrm{m}_{\mathrm{i}}\right)} * \frac{\mathrm{p}\left(\sim \mathrm{m}_{\mathrm{i}} \mid \mathrm{z}_{1: \mathrm{t}-1}, \mathrm{x}_{1: \mathrm{t}-1}\right)}{\mathrm{p}\left(\mathrm{z}_{\mathrm{t}} \mid \mathrm{z}_{1: \mathrm{t}-1}, \mathrm{x}_{1: \mathrm{t}}\right)} .
\end{gathered}
$$

The ratio of the two can be obtained by taking the logarithm.

$$
\begin{gathered}
\mathrm{l}\left(\mathrm{m}_{\mathrm{i}} \mid \mathrm{z}_{1: \mathrm{t}}, \mathrm{x}_{1: \mathrm{t}}\right)=\mathrm{l}\left(\mathrm{m}_{\mathrm{i}} \mid \mathrm{z}_{\mathrm{t}}, \mathrm{x}_{\mathrm{t}}\right)+\mathrm{l}\left(\mathrm{m}_{\mathrm{i}} \mid \mathrm{z}_{1: \mathrm{t}-1}, \mathrm{x}_{1: \mathrm{t}-1}\right)- \\
\mathrm{l}\left(\mathrm{m}_{\mathrm{i}}\right)(14)
\end{gathered}
$$

In the equation,$l\left(m_{i} \mid z_{t}, x_{t}\right)$ refers to the logarithm of the inverse observation model of LiDAR, $\mathrm{l}\left(m_{i} \mid z_{1: t-1}, x_{1: t-1}\right)$ refers to the logarithm of the state of the grid $m_{i}$ at time $t-1$, and $l\left(m_{i}\right)$ refers to the logarithm of the a priori value for the grid. After the above transformation, map updating becomes a simple addition and subtraction process, which facilitates map management.

Because of the independence of grids in maps, the map $m$ can be expressed as

$$
\mathrm{p}(\mathrm{m})=\prod_{i=1}^{i=n} \mathrm{p}\left(m_{i}\right)
$$

The process of map model construction is shown in Figure 5. The motion model "Motion" is obtained from the estimated pose $x_{t-1}$ at time $t-1$ and the odometer information $u_{t}$ at time $t$, and $x_{t}$ is derived. The observation model "Observation" is obtained from the measurement data $z_{t}$ from the LIDAR at time $t$ and the map at time $t-1$. The observation model is combined with the motion model to derive the map model at time $t$.

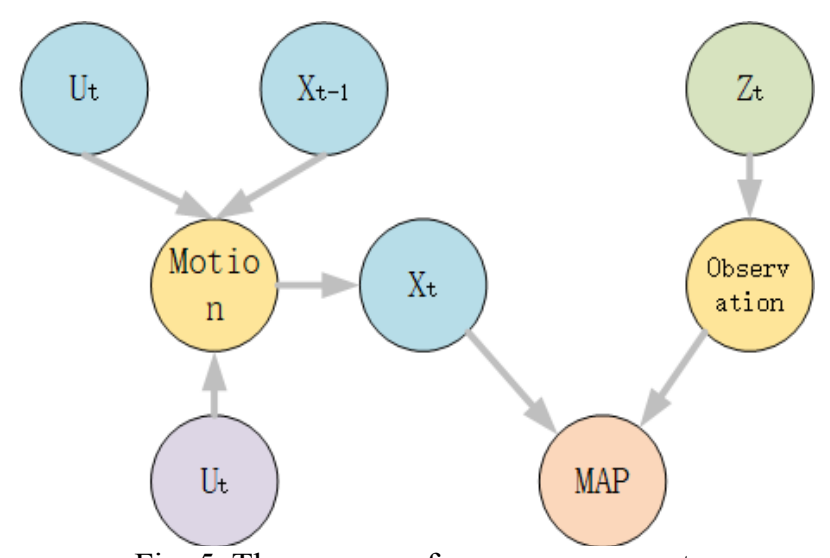

Fig. 5. The process of map management

\section{EXPERIMENT AND RESULT ANALYSIS}

$\mathrm{T}$ his experiment was tested under the ROS indigo operating system. The robot uses a Kobuki mobile chassis equipped with the UST-10LX 2D laser scanner from HOKUYO. Using this experimental platform to collect the laboratory dataset, the experimental results are directly viewed using the Rviz graphical tool under ROS. The results verify the application of the ICP algorithm in the construction and positioning of the laboratory service robot in the laboratory environment.

The ROS system is designed for robot software development, with independent programming language, node communication, and visualization tools. It abstracts sensors, controllers, and motion planning into nodes, and nodes can communicate by publishing subscription topics. So it fully satisfies the requirements of this experiment.

The UST-10LX 2D laser scanner has a measuring range of 10 meters and $270^{\circ}$. The measuring accuracy is \pm $40 \mathrm{~mm}$, the angular resolution is $0.25^{\circ}$, and the input noise is $<=25 \mathrm{~dB}$. Thus, the laboratory service robot has a good sense of the outside world.

The environment used in the experiment was the engineering building Test Technology Laboratory of Beijing Forestry University, including the laboratory corridor and the test bench area in the laboratory. It is a normal laboratory environment, with structural and non-structural characteristics, providing a wealth of experimental characteristics for this study.

\section{A. ICP algorithm matching results}

To verify if the ICP algorithm met the accuracy of the experimental results, this study simulated the algorithm firstly. Since the number of particles of laser data per frame after filtering in this experiment is between 500 and 1000 , we performed two simulations, with 500 and 1000 particles randomly placed on the plane as data $(t-1)$. These are both rotated by $10^{\circ}$, followed by a 0.5 $\mathrm{m}$ translation along the $\mathrm{X}$-axis to obtain data $(t)$. Then the ICP algorithm is used to match data $(t-1)$ and data $(t)$ in both simulations, and the matching result of data $(t)$ is obtained. Figure 6 shows the results of the ICP algorithm matching, where the blue particles are the data $(t-1)$ point cloud data, the green particles are the data $(t)$ point cloud data, and the red particles are the ICP algorithm matching results. Table 1 shows the matching result error. As the number of particles increases, the number of matching iterations will increase accordingly, and the error will decrease. That is, as the number of particles in each frame of cloud data increases, the real-time effect of the ICP algorithm will decrease and the accuracy will increase. The ICP algorithm should be determined to meet the experimental requirements. In this experiment, the ICP algorithm can meet the experimental requirements. 


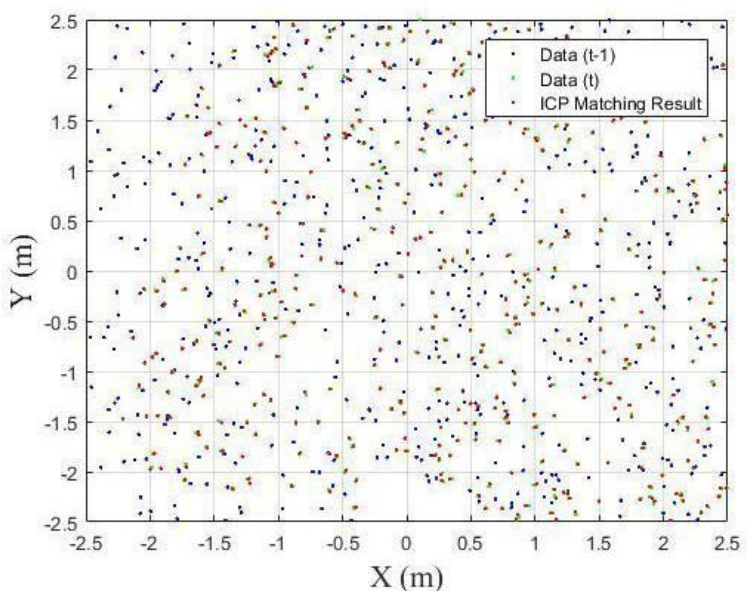

(a) Number of particles $=500$

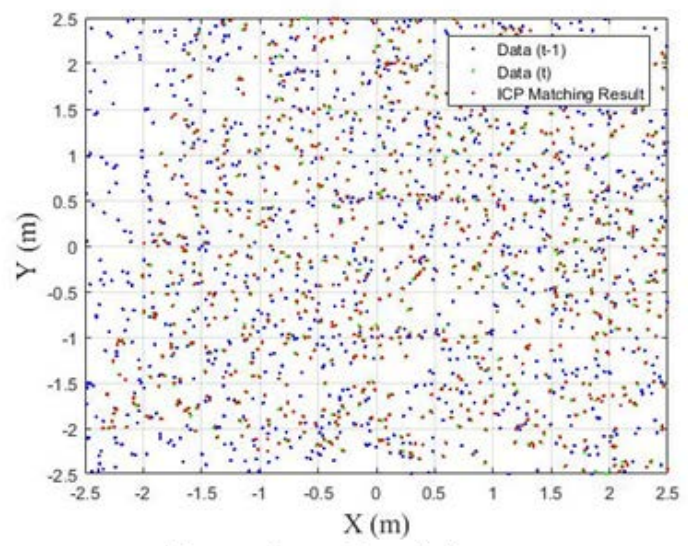

(b) Number of particles $=1000$

Fig. 6. ICP matching results

Table I

ICP matching result error

\begin{tabular}{lllll}
\hline $\begin{array}{l}\text { Number of } \\
\text { particles }\end{array}$ & $\begin{array}{l}\text { Number of } \\
\text { iterations }\end{array}$ & $\begin{array}{l}\text { X-axis } \\
\text { error }(\mathrm{m})\end{array}$ & $\begin{array}{l}\text { Y-axis } \\
\text { error }(\mathrm{m})\end{array}$ & $\begin{array}{l}\text { Angle error } \\
\text { (degrees) }\end{array}$ \\
\hline 500 & 31 & -0.0006 & 0.0004 & 0.9661 \\
1000 & 44 & -0.0003 & -0.0003 & 0.3553 \\
\hline
\end{tabular}

\section{B. Map Construction}

In this session, the robot walked through the laboratory corridor and inside the laboratory at a speed of $0.5 \mathrm{~m} / \mathrm{s}$ under remote control, and recorded data sets using an ROS bag, and verified in ROS Rviz. The node diagram of the mapping process is shown in Figure 7. After the odometer message and radar message are subscribed by ROS master, ROS master publishers the message to ICP-SLAM. After a s eries of processing, such as coordinate change, map management and pose prediction, the map information and robot pose are obtained and published.

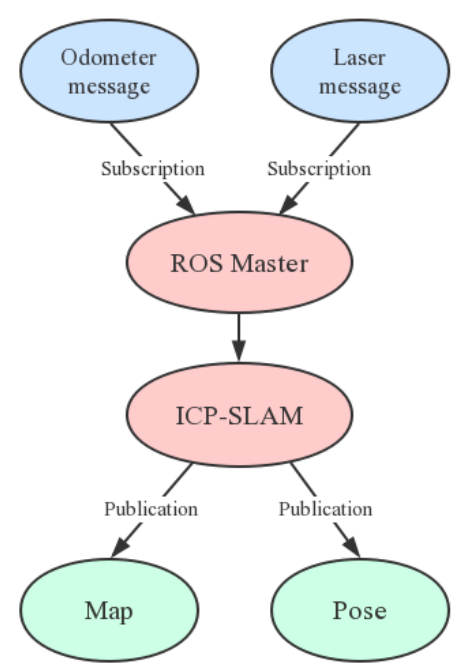

Fig. 7. Node diagram of the mapping process

Figure 8 and Figure 9 show the construction of the map in the experiment, in which the black area represents the presence of an obstacle (occupied state), the white area represents that no obstacle is present (idle state), and the gray area is the area unknown to the robot (unknown state). Figure 8 is a map construction of the laboratory corridor environment, in which obstacles are displayed on both sides of the corridor, and obstacles can be better identified on the constructed map. Figure 9 shows the construction of the internal map of the laboratory, and it can be seen that it is better to distinguish the placement of the experimental table and to identify it on the map.

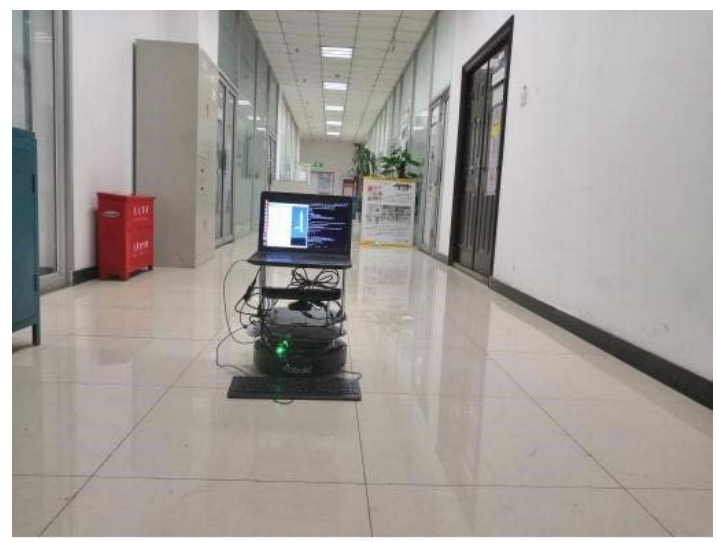

(a)

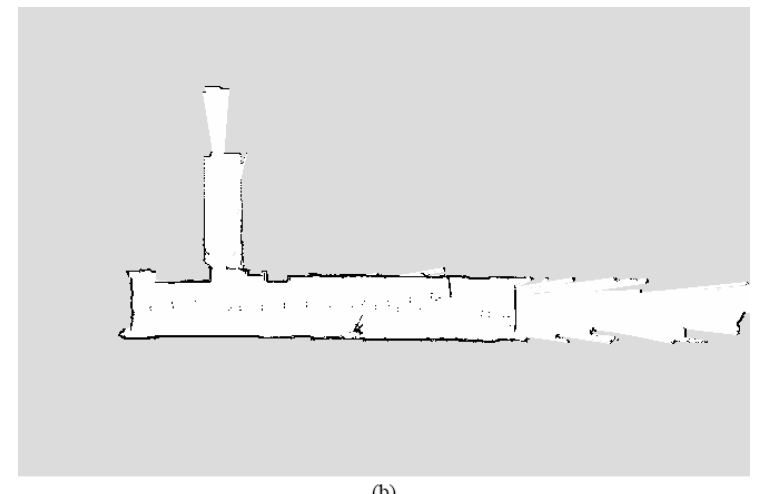

Fig. 8. Construction of the corridor map 


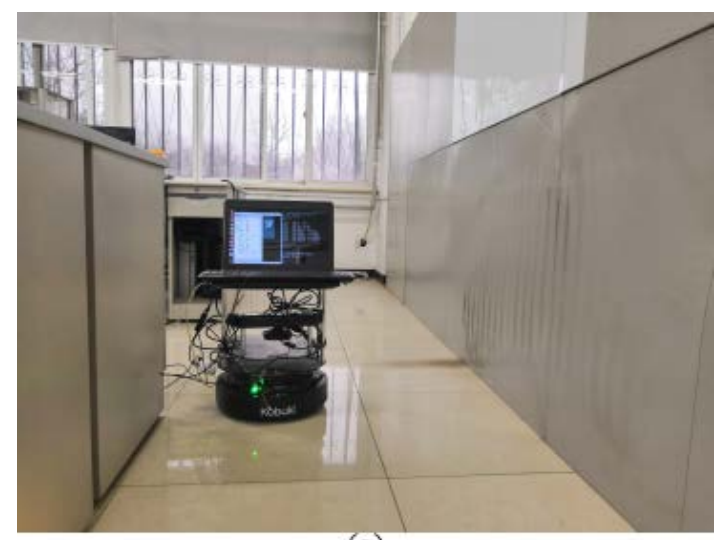

(a)

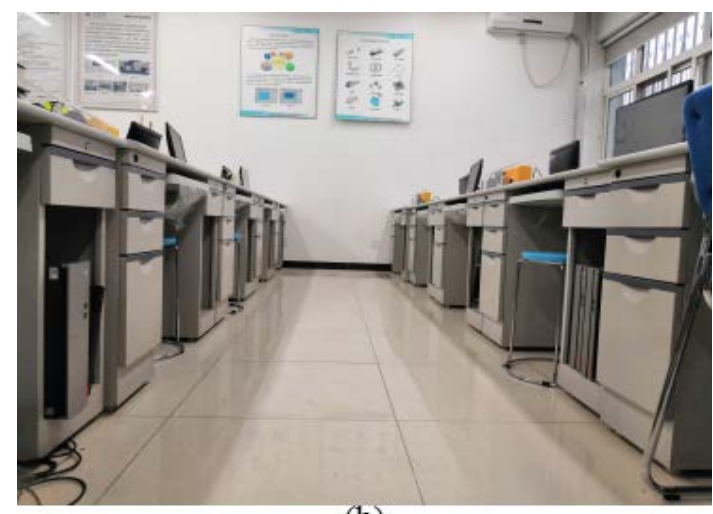

(b)

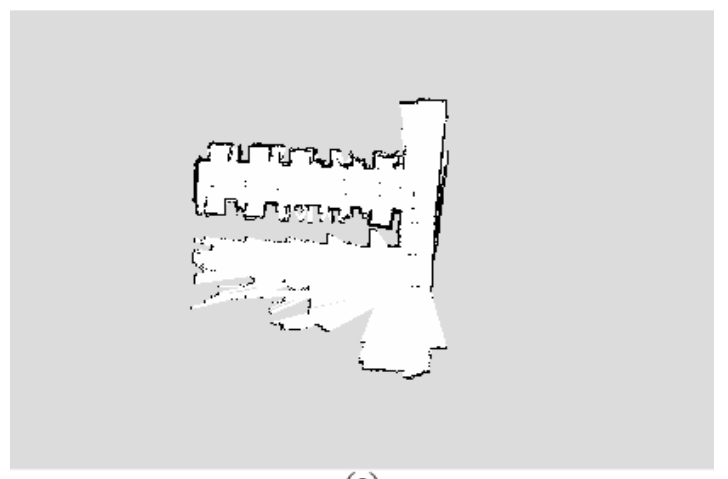

(c)

Fig. 9. Construction of the internal map of the laboratory

\section{Robot positioning verification}

The experimental platform stayed at the corner of the middle aisle of the lab table, and the coordinate system of each module of the robot was displayed in the corresponding ROS Rviz graphical interface, which fully demonstrated the posture of the experimental platform at this time. Figure 10 shows the positioning of the robot during the experiment. (a) is the actual position of the robot in the laboratory. (b) is the simulation image in Rviz, where the red circle is the location of the coordinate system of the robot. From the results, we can see that the position of the robot on the constructed map has a small error compared to the actual location, and meets the basic positioning needs of self-positioning of laboratory service robot in laboratory management.

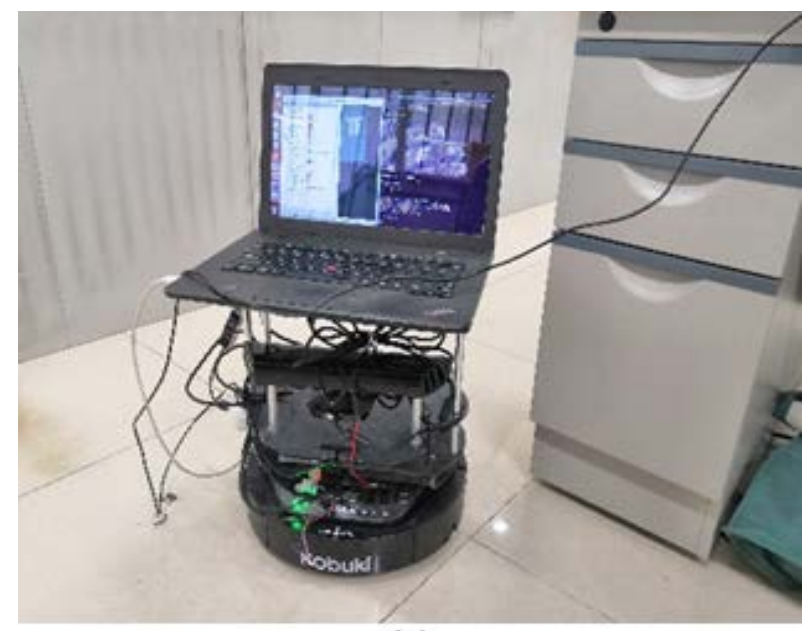

(a)

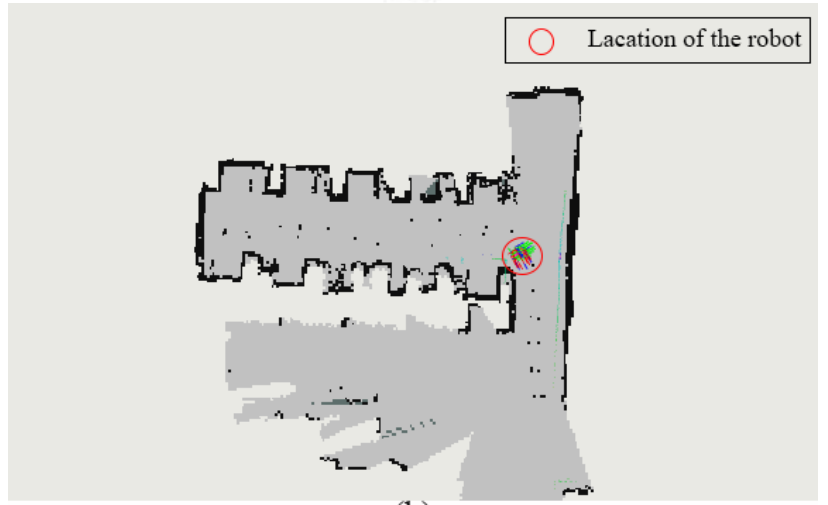

(b)

Fig. 10. Robot positioning based on ICP matching

\section{CONCLUSION AND DisCUSSION}

$\mathrm{T}$ his study builds a laboratory service robot platform with the two goals of map construction and self-positioning in the laboratory and verifies the role of the ICP algorithm in this process. Experiments show that by scanning and matching the laser points with the ICP algorithm, the robot can realize its perception of the laboratory environment, establish a laboratory map, and estimate its own position as well. This has far-reaching significance for the use of robots in the field of experimental teaching and in scientific research experiments. However, in the study, the movement of personnel in the laboratory was not considered, and dynamic objects were not processed. These factors would cause interference points to be present in the experiment. Therefore, the identification of walking personnel will be taken into account in the next study. In addition, the service robots should have good human-machine interaction capabilities, which is also the next research focus.

\section{REFERENCES}

[1] X. Zhao, J. Kan, C. Zhan. "1-Point RANSAC Application: An Independent Positioning Method for Crawler Mobile Robots Based on Binocular Stereo Vision," International Journal of Circuits, Systems and Signal Processing, vol. 12, pp. 105-111, 2018.

[2] D M. A.-Latif, M A.-Megeed Salem, H. Ramadan, M. I. Roushdy. "3D Graph-based Vision-SLAM Registration and Optimization," International Journal of Circuits, Systems and Signal Processing, vol. 8, pp. 123-130, 2014 
[3] S. F. Wei, F. Pang, Z. B. Liu, X. J. Shi. "Survey of lidar-based SLAM algorithms," Application Research of Computers, vol. 37, no. 2, 2018.

[4] W. T. Gong, Z. L. Sun, P. Pan. "Indoor mapping of robot based on 2D laser," Microcomputer \& Its Applications, vol. 18, pp. 55-58, 2017.

[5] P. J. Besl, Member, IEEE, N. D. Mckay. "A method for registration of 3-D shapes," IEEE Trans. Pattern Anal. Mach. Intell, vol.14, no.2, pp.239-256, 1992.

[6] D. Holz, S. Behnke. "Sancta Simplicitas - On the efficiency and achievable results of SLAM using ICP-Based Incremental Registration," presented at IEEE International Conference on Robotics and Automation., Anchorage, USA, June, 2010.

[7] S. Y. Peng. "The SLAM Research of Indoor Robot Based on Laser Range Finder", Master's thesis, Wuhan, Wuhan University Of Science and Technology,2012.

[8] R. Tiar, N. Ouadah, O. Azouaoui. "ICP-SLAM methods implementation on a bi-steerable mobile robot," presented at 2013 IEEE 11th International Workshop of. IEEE, June, 2013.

[9] W. Gu, B. Zhou, X. Dai. "ICP matching algorithm-based localization of indoor mobile robots," Journal of Huazhong University of Science \& Technology (Natural Science Edition), vol. 41, pp. 262-266, 2013.

[10] M. Djehaich, H. Ziane, N. Achour. "SLAM-ICP with a Boolean method applied on a c ar-like robot," presented at Programming and Systems (ISPS), 2013 11th International Symposium on IEEE, April, 2013.

[11] J. Liu, L. Li. "A scanning matching method based on laser range finder," Engineering of Surveying and Mapping, vol. 5, pp.64-71, 2018.

[12] H. Cho, E. K. Kim, S. Kim. "Indoor SLAM application using geometric and ICP matching methods based on line features," Robotics and Autonomous Systems, vol. 100, pp. 206-224, 2018.

[13] J. Yang, L. Sun, Y. Shao. "Efficient Iterative Closest Point Localisation Algorithm for Autonomous Robots," Journal of Mechanical Engineering, vol. 54, no. 15, pp.15-20, 2018.

[14] B. Silvère, M. Barczyk, F. Goulette. "On the Covariance of ICP-based Scan-matching Techniques," Computer Science, pp. 5498-5503, 2016.

[15] Z. Zhang. Iterative Closest Point (ICP). Computer Vision, Springer US, 2014, pp.433-434.

[16] C. Chen, Y. Qi, Y. Zhu, et al. "The Analysis of Influencing Factors and Evaluation Indexes On ICP Algorithm," Navigation positioning \& Timing, vol. 5, no.5, pp.67-72, 2018.

[17] P. Bergström, O. Edlund. "Robust registration of point sets using iteratively reweighted least squares" Computational Optimization \& Applications, vol. 58, no. 3, pp. 543-561, 2014.

[18] S. Zhao. "Research and Implementation of Mobile Robot SLAM Using Low Cost Laser Sensor", Master's thesis, Jinan, Shandong University, 2017.

[19] X. Ren. "Lidar Indoor SLAM Method", Master's thesis, Harbin, Harbin Engineering University, 2018.
Jian Wu, born in 1989, studied in Beijing Forestry University at 2006 2013, received his Master degree in mechanical manufacturing and automation from Beijing Forestry University in 2013. Now he works in the internship and experiment center of the school of technology in Beijing Forestry University. $\mathrm{He}$ is interested in researching intelligent control and intelligent detection technology.

Ruting Yao, born in 1995, received her Bachelor's degree from Qingdao University of Technology in 2018. Since September of 2018, she has been studying for her postgraduate degree in Beijing Forestry University. Her research interests include forestry robotics and information fusion.

Yili Zheng, born in 1981, is an associate professor at Beijing Forestry University. In 2009, he received his Doctor's degree from Beijing University of Posts and Telecommunications. His research interests include forestry informatization and forestry engineering equipment.

Jinhao Liu, born in 1958, is a professor at Beijing Forestry University. He received his Doctor's degree in Northeast Forestry University. He is interested in researching automation and intelligence of forestry equipment. 